\title{
Upper Bounds for Mutations of Potentials ${ }^{\star}$
}

\author{
John Alexander CRUZ MORALES $\dagger^{1}$ and Sergey GALKIN $\dagger^{2} \dagger^{3} \dagger^{4} \dagger^{5}$ \\ $\dagger^{1}$ Department of Mathematics and Information Sciences, Tokyo Metropolitan University, \\ Minami-Ohsawa 1-1, Hachioji, Tokyo 192-037, Japan \\ E-mail: cruzmorales-johnalexander@ed.tmu.ac.jp, alekosandro@gmail.com \\ $\dagger^{2}$ Kavli Institute for the Physics and Mathematics of the Universe, The University of Tokyo, \\ 5-1-5 Kashiwanoha, Kashiwa, 27r-8583, Japan \\ $\dagger^{3}$ Independent University of Moscow, 11 Bolshoy Vlasyevskiy per., 119002, Moscow, Russia \\ $\dagger^{4}$ Moscow Institute of Physics and Technology, 9 Institutskii per., \\ Dolgoprudny, 141700, Moscow Region, Russia \\ E-mail: Sergey.Galkin@phystech.edu \\ $\dagger^{5}$ Universität Wien, Fakultät für Mathematik, Garnisongasse 3/14, A-1090 Wien, Austria
}

Received May 31, 2012, in final form January 16, 2013; Published online January 19, 2013

http://dx.doi.org/10.3842/SIGMA.2013.005

\begin{abstract}
In this note we provide a new, algebraic proof of the excessive Laurent phenomenon for mutations of potentials (in the sense of [Galkin S., Usnich A., Preprint IPMU 10-0100, 2010]) by introducing to this theory the analogue of the upper bounds from [Berenstein A., Fomin S., Zelevinsky A., Duke Math. J. 126 (2005), 1-52].
\end{abstract}

Key words: cluster algebras; Laurent phenomenon; mutation of potentials; mirror symmetry

2010 Mathematics Subject Classification: 13F60; 14J33; 53D37

\section{Introduction}

The idea of mutations of potentials was introduced in [9] and the Laurent phenomenon was established in the two dimensional case by means of birational geometry of surfaces. More precisely, in op. cit. the authors considered a toric surface $X$ with a rational function $W$ (a potential), and using certain special birational transformations (mutations), they established the (excessive) Laurent phenomenon which roughly says that if $W$ is a Laurent polynomial whose mutations are Laurent polynomials, then all subsequent mutations of these polynomials are also Laurent polynomials (see Theorem B.1 in Appendix B for a precise statement of the excessive Laurent phenomenon as established in [9]). The motivating examples of such potentials come from the mirror images of special Lagrangian tori on del Pezzo surfaces [8] and Auroux's wall-crossing formula relating invariants of different tori [2].

The cluster algebras theory of Fomin and Zelevinsky [7] provides an inductive way to construct some birational transformations of $n$ variables as a consecutive composition of elementary ones (called elementary mutations) with a choice of $N=n$ directions at each step.

The theory developed in [9] can be seen as an extension of the theory of cluster algebras [7] when the number of directions of mutations $N$ is allowed to be (much) bigger than the number of variables $n$, but at least one function remains to be a Laurent polynomial after all mutations. So, it is natural to try to extend the machinery of the theory of cluster algebras for this new setup. The main goal of this paper is to give the first step in such an extension by means of

\footnotetext{
*This paper is a contribution to the Special Issue "Mirror Symmetry and Related Topics". The full collection is available at http://www.emis.de/journals/SIGMA/mirror_symmetry.html
} 
the introduction of the upper bounds (in the sense of [3]) and establishing the excessive Laurent phenomenon [9] in terms of them. It is worth noticing that a further generalization can be done and in a forthcoming work [5] we plan to study the quantization of the mutations of potentials and their upper bounds. Naturally, this quantization can be seen as an extension of the theory of quantum cluster algebras developed in $[4,11]$ and the theory of cluster ensembles in [6].

The upper bounds introduced in this paper can be described as a collection of regular functions that remain regular after one elementary mutation in any direction. Thus, we can establish the main result of this paper in the following terms (see Theorem 3.1 for the exact formulation).

Theorem (Laurent phenomenon in terms of the upper bounds). The upper bounds are preserved by mutations.

Aside from providing a new proof for the excessive Laurent phenomenon and the already mentioned generalization in the quantized setup, the algebraic approach that we are introducing here is helpful for tackling the following two problems:

1. Develop a higher dimensional theory (i.e. dimension higher than 2) for the mutations of potentials. Some work in that direction is carried out in [1].

2. Present an explicit construction to compactify Landau-Ginzburg models (Problem 44 of [9]).

In the present paper we do not deal with the above two problems (only a small comment on 2 will be made at the end of the paper). We plan to give a detailed discussion of them in [5] too. We just want to mention that the new algebraic approach has interesting geometrical applications.

Some words about the organization of the text are in order. In Section 2 we extend the theory developed in [9] to lattices of arbitrary rank and general bilinear forms (i.e., we can consider even degenerate and not unimodular forms) and introduce the notion of upper bounds in order to establish our main theorem. In Section 3 we actually establish the main theorem and present its proof when the rank of the lattice is two and the form is non-degenerate which is the case of interest for the geometrical setup of [9]. In the last section some questions and future developments are proposed. For the sake of completeness of the presentation we include two appendices. In Appendix A we review some definitions of [3] and briefly compare their theory with ours. Appendix B is dedicated to presenting the Laurent phenomenon in terms of [9].

\section{Mutations of potentials and upper bounds}

Now we present an extension of the theory of mutations of potentials [9] (as formulated by the second author and Alexandr Usnich) and introduce our modified definitions with the new definition of upper bound. Notice that a slightly different theory (which fits into the framework of this paper, but not [9]) is used in our software code $^{1}$.

\subsection{Combinatorial data}

Let $(\cdot, \cdot): L^{*} \times L \rightarrow \mathbb{Z}$ be the canonical pairing between a pair of dual lattices $L \simeq \mathbb{Z}^{r}$ and $L^{*}=\operatorname{Hom}(L, \mathbb{Z}) \simeq \mathbb{Z}^{r}$.

In what follows the lattice $L$ is endowed with a skew-symmetric bilinear integral form $\omega$ : $L \times L \rightarrow \mathbb{Z}$ (we use the notation $\left\langle v, v^{\prime}\right\rangle=\omega\left(v, v^{\prime}\right)$ ). In the most important (both technically, and from the point of view of applications) case $r=\operatorname{rank} L=2$, we have $\Lambda^{2} L \simeq \mathbb{Z}$, so all integer skew-symmetric bilinear forms are integer multiples $\omega_{k}=k \omega_{1}(k \in \mathbb{Z})$ where a generator $\omega_{1}$ is

\footnotetext{
${ }^{1}$ http://member .ipmu.jp/sergey.galkin/degmir.gp.
} 
fixed by the choice of orientation on $L \otimes \mathbb{R}$ so that $\omega_{1}((1,0),(0,1))=1$. We would occasionally use notations $\langle\cdot, \cdot\rangle_{1}=\omega_{1}(\cdot, \cdot)$ and $\langle\cdot, \cdot\rangle_{k}=\omega_{k}(\cdot, \cdot)$.

The bilinear form $\omega$ gives rise to a map $i=i_{\omega}: L \rightarrow L^{*}$ that sends an element $v \in L$ into a linear form $i_{\omega}(v) \in L^{*}$ such that $\left(i_{\omega}(v), v^{\prime}\right)=\omega\left(v, v^{\prime}\right)$ for any $v^{\prime} \in L$. The map $i_{\omega}$ is an isomorphism $\Longleftrightarrow$ the form $\omega$ is non-degenerate and unimodular, when $\omega$ is non-degenerate but not unimodular the map $i$ identifies the lattice $L$ with a full sublattice in $L^{*}$ of index $\operatorname{det} \omega$, finally if $\omega$ is degenerate then both the kernel and the cokernel of the map $i_{\omega}$ has positive rank.

We would like to have some functoriality, so we consider a category whose objects are given by pairs $(L, \omega)$ of the lattice $L$ and a skew-symmetric bilinear form $\omega$, and the morphisms $\operatorname{Hom}\left(\left(L^{\prime}, \omega^{\prime}\right),(L, \omega)\right)$ are linear maps $f: L^{\prime} \rightarrow L$ such that $\omega^{\prime}=f^{*} \omega$, i.e. $\omega\left(v_{1}, v_{2}\right)=$ $\omega^{\prime}\left(f\left(v_{1}\right), f\left(v_{2}\right)\right)$ for all $v_{1}, v_{2} \in L^{\prime}$. Any linear map $f: L^{\prime} \rightarrow L$ defines an adjoint $f^{*}: L^{*} \rightarrow L^{\prime *}$ and if it respects the bilinear forms, then $i_{\omega^{\prime}}=f^{*} i_{\omega} f$.

For a vector $u \in L$ we define a symplectic reflection $R_{u}$ and a piecewise linear mutation $\mu_{u}$ to be the (piecewise)linear automorphisms of the set $L$ given by the formulae

$$
\begin{aligned}
& R_{\omega, u}(v)=v+\omega(u, v) u, \\
& \mu_{\omega, u} v=v+\max (0, \omega(u, v)) u .
\end{aligned}
$$

For any morphism $f \in \operatorname{Hom}\left(\left(L^{\prime}, \omega^{\prime}\right),(L, \omega)\right)$ and any vector $u \in L^{\prime}$ we have $R_{\omega, f u} f=f R_{\omega^{\prime}, u}$ and $\mu_{\omega, f u} f=f \mu_{\omega^{\prime}, u}$. Indeed, $f \mu_{u} v=f\left(v+\max \left(0, \omega^{\prime}(u, v)\right) u\right)=f v+\max \left(0, \omega^{\prime}(u, v)\right)(f u)=$ $f v+\max (0, \omega(f u, f v))(f u)=\mu_{f u}(f v)$.

Note that $R_{a \omega, b u}=R_{\omega, u}^{a b^{2}}$ for all $a, b \in \mathbb{Z}$ and $\mu_{a \omega, b u}=\mu_{\omega, u}^{a b^{2}}$ for all $a, b \in \mathbb{Z}_{+}$. However $\mu_{\omega,-u} v=$ $-\mu_{u}(-v)=v+\min (0, \omega(u, v)) u$, hence $\mu_{\omega,-u} \mu_{\omega, u}=R_{\omega, u}$. Both $R_{\omega, u}$ and $\mu_{\omega, u}$ are invertible: $R_{\omega, u}^{-1} v=R_{-\omega, u} v=v-\omega(u, v) u, \mu_{\omega, u}^{-1} v=\mu_{-\omega,-u} v=R_{\omega, u}^{-1} \mu_{\omega,-u} v=v-\max (0, \omega(u, v)) u$. Note that $\mu_{\omega,-u}^{-1} v=R_{\omega,-u}^{-1} \mu_{\omega, u} v=R_{\omega, u}^{-1} \mu_{\omega, u}(v)=v-\min (0, \omega(u, v)) u$. Therefore, changing $\max$ by min and + by - , simultaneously, corresponds to changing the form $\omega$ to the opposite $-\omega$. Further we omit $\omega$ from the notations of $R_{u}$ and $\mu_{u}$ where the choice of the form is clear.

The underlying combinatorial gadget of our story is a collection of $n$ vectors in $L$ :

Definition 2.1. An exchange collection $V$ is an element of $L^{n}$, i.e. an $n$-tuple $\left(v_{1}, \ldots, v_{n}\right)$ of vectors $v_{i} \in L$. Some $v_{i}$ may coincide. For a vector $v$ its multiplicity $m_{V}(v)$ in the exchange collection $V$ equals the number of vectors in $V$ that coincide with $v: m_{V}(v)=\#\{1 \leqslant i \leqslant n$ : $\left.v_{i}=v\right\}$. We say that an exchange collection $V^{\prime}$ is a subcollection of exchange collection $V$ if $m_{V^{\prime}} \leqslant m_{V}$. Equivalently, one may define an exchange collection $V$ by its (non-negative integer) multiplicity function $m_{V}: L \rightarrow \mathbb{Z}_{\geqslant 0}$. In this case $n=\sum_{v \in L} m_{V}(v)$.

The exchange collections could be pushed forward by morphisms $f \in \operatorname{Hom}\left(\left(L^{\prime}, \omega^{\prime}\right),(L, \omega)\right)$ : $v_{1}^{\prime}, \ldots, v_{n}^{\prime} \in L^{\prime n}$ will go to $f v_{1}, \ldots, f v_{n} \in L^{n}$. This gives rise to a natural diagonal action of $\operatorname{Aut}(L, \omega)=\operatorname{Sp}(L, \omega)$ on $L^{n}$. This action commutes with the permuting action of $S_{n}$.

A vector $n \in L$ is called primitive if it is nonzero and its coordinates are coprime, i.e. $n$ does not belong to the sublattice $k L$ for any $k>1$, in other words $n$ is not a multiple of other vector in $L$. We denote the set of all primitive vectors in $L$ as $L_{1}$. Similarly one can define primitive vectors in the dual lattice $L^{*}$. Note that if $\operatorname{det} \omega \neq \pm 1$ then $i_{\omega}(n)$ may be a non-primitive element of $L^{*}$ even for primitive elements $n \in L_{1}$.

\subsection{Birational transformations}

Consider the group ring $\mathbb{Z}\left[L^{*}\right]$ - ring of Laurent polynomials of $r$ variables. Its spectrum $T=$ Spec $\mathbb{Z}\left[L^{*}\right] \simeq \mathrm{G}_{m}^{r}(\mathbb{Z})$ is the $r$-dimensional torus over the integers, in particular $T(\mathbb{C})=$ $\operatorname{Hom}\left(L^{*}, \mathbb{C}^{*}\right), L^{*}=\operatorname{Hom}\left(T, \mathrm{G}_{m}\right)$ is the lattice of characters of $T$ and $L=\operatorname{Hom}\left(\mathrm{G}_{m}, T\right)$ is the 
lattice of 1-parameter subgroups in $T$. Define the ambient field $\mathbb{K}=\mathbb{K}_{L}=\mathcal{Q}\left(L^{*}\right)$ as the fraction field of $\mathbb{Z}\left[L^{*}\right]$ extended by all roots of unity $(\mathcal{Q}=\mathbb{Q}(\exp (2 \pi i \mathbb{Q}))$.

A vector $u \in L$ defines a birational transformation of $\mathbb{K}_{L}$ (and its various subfields and subrings) as follows

$$
\mu_{u, \omega}: X^{m} \rightarrow X^{m}\left(1+X^{i_{\omega}(u)}\right)^{(u, m)} .
$$

If $f: T_{1} \rightarrow T_{2}$ is a rational map between two tori, and $u: \mathrm{G}_{m} \rightarrow T_{1}$ is a one-parameter subgroup of $T$ then its image $f u: \mathrm{G}_{m} \rightarrow T_{2}$ is not necessarily a one-parameter subgroup, but asymptotically behaves like one, this defines a tropicalization map $T(F): \operatorname{Hom}\left(\mathrm{G}_{m}, T_{1}\right) \rightarrow$ $\operatorname{Hom}\left(\mathrm{G}_{m}, T_{2}\right)$. The tropicalization of the birational map $\mu_{u, \omega}: T_{1} \rightarrow T_{2}$ is the piecewise-linear map $\mu_{u, \omega}: L_{1} \rightarrow L_{2}$ defined in the previous subsection.

One can easily see most of the relations of the previous subsection on the birational level. For example, $\mu_{-u} \mu_{u}=\mu_{u} \mu_{-u}=R_{u}$ and $R_{v} \mu_{u} R_{v}^{-1}=\mu_{R_{v} u}$, where $R_{u}$ is the homomorphism of the torus $T$ given by $R_{u, \omega}: X^{m} \rightarrow X^{m+(u, m) i_{\omega} u}$. Also $R_{a u, b \omega}=R_{u, \omega}^{a^{2} b}$ for any $a, b \in \mathbb{Z}$, and $\mu_{a u, \omega}=\left(\mu_{u, a \omega}\right)^{a}$ for any $a \in \mathbb{Z}$, however neither of them is a power of $\mu_{u, \omega} \cdot{ }^{2}$ In particular, $\left(\mu_{u, \omega}\right)^{-1}=\mu_{-u,-\omega}$.

Note that if $M \subset L^{*}$ is some sublattice of $L^{*}$ that contains $i_{\omega}(u)$ then $\mu_{u}$ preserves the fraction field of $\mathbb{Z}[M] \subset \mathbb{Z}\left[L^{*}\right]$. For any morphism $f \in \operatorname{Hom}\left(\left(L^{\prime}, \omega^{\prime}\right),(L, \omega)\right)$ and a vector $u \in L^{\prime}$ we have a homomorphism $f^{*}: \mathbb{Z}\left[L^{*}\right] \rightarrow \mathbb{Z}\left[L^{\prime *}\right]$ and two birational transformations $\mu_{u} \in$ Aut $\mathbb{K}_{L^{\prime}}, \mu_{f u} \in$ Aut $\mathbb{K}_{L}$ that commute: $\mu_{u} f^{*}=f^{*} \mu_{f u}$.

Remark 2.1. We have the following functoriality of the mutations with respect to the lattice $L$ : let $L^{\prime} \subset L$ be a sublattice of index $k$ in the lattice $L$, so $L^{*}=\operatorname{Hom}(L, \mathbb{Z})$ is a sublattice of index $k$ in $L^{\prime *}=\operatorname{Hom}\left(L^{\prime}, \mathbb{Z}\right)$, and assume that the vector $u$ lies in the sublattice $L^{\prime}$. Then the Abelian group $G=\left(L / L^{\prime}\right)$ of order $k$ acts on $\mathcal{Q}\left[L^{\prime *}\right],{ }^{3}$ and its invariants is the subring $\mathcal{Q}\left[L^{*}\right]$, so $G$ acts on the torus $T^{\prime}=\operatorname{Spec} \mathcal{Q}\left[L^{\prime *}\right]$ and the torus $T=\operatorname{Spec} \mathcal{Q}\left[L^{*}\right]$ is the quotient-torus $T=T^{\prime} / G$, let $\pi: T^{\prime} \rightarrow T$ be the projection to the quotient. The vector $u$ defines the birational transformation $\mu_{u, T}$ of the torus $T$ and the birational transformation $\mu_{u, T^{\prime}}$ of the torus $T^{\prime}$. Then the mutation $\mu_{u}$ commutes with the action of the group $G$ and with the projections: $\pi \mu_{u, T^{\prime}}=\mu_{u, T} \pi$ and $g \mu_{u, T^{\prime}}=\mu_{u, T^{\prime}} g$ for any $g \in G$.

\subsubsection{Rank two case}

Let us see the mutations explicitly in case rank $L=2$. Let $e_{1}, e_{2}$ be a base of $L$ and $f_{1}, f_{2}$ be the dual base of $L^{*}$, so $\left(e_{i}, f_{j}\right)=\delta_{i, j}$. Also let $x_{i}=X^{f_{i}}$ be the respective monomials in $\mathbb{Z}\left[L^{*}\right]$. For the skew-symmetric bilinear form $\omega_{k}$ defined by $\omega_{k}\left(e_{1}, e_{2}\right)=k$ and a vector $u=u_{1} e_{1}+u_{2} e_{2} \in L$ we have $i_{\omega_{k}}\left(u_{1} e_{1}+u_{2} e_{2}\right)=\left(-k u_{2}\right) f_{1}+\left(k u_{1}\right) f_{2}$ and so

$$
\mu_{u, \omega_{k}}:\left(x_{1}, x_{2}\right) \rightarrow\left(x_{1} \cdot\left(1+x_{1}^{-k u_{2}} x_{2}^{k u_{1}}\right)^{u_{1}}, x_{2} \cdot\left(1+x_{1}^{-k u_{2}} x_{2}^{k u_{1}}\right)^{u_{2}}\right),
$$

in particular the inverse map to $\mu_{u, \omega_{1}}$ is given by $\mu_{-u,-\omega_{1}}:\left(x_{1}, x_{2}\right) \rightarrow\left(x_{1} \cdot\left(1+x_{1}^{u_{2}} x_{2}^{-u_{1}}\right)^{-u_{1}}, x_{2}\right.$. $\left.\left(1+x_{1}^{u_{2}} x_{2}^{-u_{1}}\right)^{-u_{2}}\right)$. In particular, $\mu_{(0,1)}^{*} f=f\left(x_{1}, \frac{x_{2}}{1+x_{1}}\right)$.

For any matrix $A=\left(\begin{array}{ll}a & b \\ c & d\end{array}\right) \in \operatorname{SL}(2, \mathbb{Z})=\operatorname{Sp}(L, \omega)$ there is a regular automorphism of the torus $t_{A}^{*}\left(x_{1}, x_{2}\right)=\left(x_{1}^{a} x_{2}^{b}, x_{1}^{c} x_{2}^{d}\right)$. Conjugation by this automorphism acts on the set of mutations: $\mu_{A u}^{*}=\left(t_{A} \mu_{u} t_{A}^{-1}\right)^{*}$. So any mutation commutes with an infinite cyclic group given by the stabilizer of $u$ in $\operatorname{Sp}(L, \omega)$, explicitly if $u=(0,1)$ then in coordinates $\left(x_{1}, x_{2}\right)$ and $\left(x_{1}, x_{2}^{\prime}=x_{1} x_{2}\right)$

\footnotetext{
${ }^{2}$ Since $\left(1+x^{a}\right)$ is not a power of $(1+x)$.

${ }^{3}$ An element $n$ in $L$ multiplies monomial $X^{m^{\prime}}$ by the root of unity $\exp \left((2 \pi i)\left(n, m^{\prime}\right)\right)$, here $\left(n, m^{\prime}\right)$ is bilinear pairing between $L$ and $L^{\prime *}$ with values in $\mathbb{Q}$ extended by linearity from the pairing $L^{\prime} \otimes L^{\prime *} \rightarrow \mathbb{Z}$.
} 
the mutation $\mu_{(0,1)}$ is given by the same formula. Also every mutation commutes with 1dimensional subtorus of $T$, in case of $u=(0,1)$ the action of the subtorus is given by $\left(x_{1}, x_{2}\right) \rightarrow$ $\left(x_{1}, \alpha x_{2}\right)$.

\subsection{Mutations of exchange collections and seeds}

Let $L$ be a lattice equipped with a bilinear skew-symmetric form $\omega$. A cluster $\mathbf{y} \in \mathbb{K}_{L}^{m}$ is a collection $\mathbf{y}=\left(y_{1}, \ldots, y_{m}\right)$ of $m$ rational functions $y_{i} \in \mathbb{K}_{L}$. We call $\mathbf{y}$ a base cluster if $\mathbf{y}=\left(y_{1}, \ldots, y_{r}\right)$ is a base of the ambient field $\mathbb{K}_{L}$. A $C$-seed (supported on $(L, \omega)$ ) is a pair $(\mathbf{y}, V)$ of a cluster $\mathbf{y} \in \mathbb{K}_{L}^{m}$ and an exchange collection $V=\left(v_{1}, \ldots, v_{n}\right) \in L^{n}$. A $V$-seed (supported on $(L, \omega))$ is a pair $(W, V)$ of a rational function $W \in \mathbb{K}_{L}$ and an exchange collection $V=\left(v_{1}, \ldots, v_{n}\right) \in L^{n}$.

Given two exchange collections $V^{\prime}=\left(v_{1}^{\prime}, \ldots, v_{n}^{\prime}\right) \in L^{\prime n}$ and $V=\left(v_{1}, \ldots, v_{n}\right) \in L^{n}$ we say that $V^{\prime}$ is a mutation of $V$ in the direction $1 \leqslant j \leqslant n$ and denote it by $V^{\prime}=\mu_{j} V$ if under the given identification $s_{j}: L \simeq L^{\prime}$ we have $v_{j}^{\prime}=s_{j}\left(-v_{j}\right)$ and $v_{i}^{\prime}=s_{j}\left(\mu_{v_{j}} v_{i}\right)$ for $i \neq k$.

The mutation of a $C$-seed $(\mathbf{y}, V)$ in the direction $1 \leqslant j \leqslant n$ is a new $C$-seed $\left(\mathbf{y}_{j}, V_{j}\right)$ where $V_{j}=\mu_{j} V$ is a mutation of the exchange collection, and $\mathbf{y}_{j}=\mu_{v_{j}, \omega} \mathbf{y}$ where each variable is transformed by the birational transformation $\mu_{v_{j}, \omega}$.

The identity $\mu_{-u} \mu_{u}=R_{u}$ implies that $\mu_{j}\left(\mu_{j}(V)\right)$ and $V$ are related by the $\operatorname{Sp}(L, \omega)$-transformation $R_{u}$.

\subsection{Upper bounds and property $(V)$}

Definition 2.2 (property $(V)$ ). We say a $V$-seed $(W, V)$ satisfies property $(V)$ if $W$ is a Laurent polynomial and for all $v \in L$ the functions $\left(\mu_{v}^{*}\right)^{m_{V}(v)} W$ are also Laurent polynomials.

In this paper we introduce the upper bound of an exchange collection.

Definition 2.3 (upper bounds). For a $C$-seed $\Sigma=(\mathbf{y}, V)$ define its upper bound $\mathcal{U}(\Sigma)$ to be the $\mathcal{Q}$-subalgebra of $\mathbb{K}_{L}$ given by

$$
\mathcal{U}(\Sigma)=\mathcal{Q}\left[\mathbf{y}^{ \pm 1}\right] \cap\left(\cap_{v \in L} \mathcal{Q}\left[\left(\mu_{v}^{*}\right)^{m_{V}(v)} \mathbf{y}^{ \pm 1}\right]\right) .
$$

In case $\mathbf{y}$ is a base cluster (by abuse of notation) we denote $\mathcal{U}(\Sigma)$ just by $\mathcal{U}(V)$.

The upper bounds defined here are a straightforward generalization of the upper bounds in [3], but also they can be thought of as the gatherings of all potentials satisfying property $(V)$.

Proposition 2.1 (relation between property $(V)$ and upper bounds). The upper bound $\mathcal{U}(V)$ of an exchange collection $V$ consists of all functions $W \in \mathbb{K}_{L}$ such that the $V$-cluster $(W, V)$ satisfies property $(V)$.

Proposition 2.2. Any morphism $f:(L, \omega) \rightarrow\left(L^{\prime}, \omega^{\prime}\right)$ induces a dual morphism $f^{*}: L^{\prime *} \rightarrow L^{*}$, a homomorphism of algebras $f^{*}: \mathbb{Z}\left[L^{\prime *}\right] \rightarrow \mathbb{Z}\left[L^{*}\right]$. Assume that this homomorphism has no kerne $l^{4}$. Then it induces a homomorphism of upper bounds $f^{*}: \mathcal{U}\left(L^{\prime}, \omega^{\prime} ; f V\right) \rightarrow \mathcal{U}(L, \omega ; V)$. In particular, if $f$ is an isomorphism, then maps $f^{*}$ and $\left(f^{-1}\right)^{*}$ establish the isomorphisms between the upper bounds $f^{*}: \mathcal{U}\left(L^{\prime}, \omega^{\prime} ; f V\right) \simeq \mathcal{U}(L, \omega ; V)$.

Proposition 2.3. Consider a seed $\Sigma=\left(L, \omega ; v_{1}, \ldots, v_{n}\right)$. For a sublattice $L^{\prime} \subset L$ that contains all vectors $v_{i} \in L^{\prime} \subset L$ consider the seed $\Sigma^{\prime}=\left(L^{\prime}, \omega_{L_{L^{\prime}}} ; v_{1}, \ldots, v_{n}\right)$. By Remark 2.1 there is a natural action of $G=L / L^{\prime}$ on $\mathbb{K}_{L^{\prime}}$ with $\mathbb{K}_{L}=\mathbb{K}_{L^{\prime}}^{G}$. Moreover, the action of $G$ obviously

\footnotetext{
${ }^{4}$ One can bypass this assumption by defining the upper bound $\mathcal{U}(L, \omega ; V)$ as a subalgebra in some localization of $\mathbb{Z}\left[L^{*}\right]$ determined by the exchange collection $V$.
} 
preserves the property of being a Laurent polynomial (i.e. it preserves the subalgebras $\mathcal{Q}\left[L^{\prime *}\right]$ ), and the mutations $\mu_{v_{i}}$ commute with the G-action. Thus the upper bound with respect to the overlattice $L$ is the subring of $G$-invariants of the upper bound with respect to the sublattice $L^{\prime}$ : $\mathcal{U}(\Sigma)=\mathcal{U}\left(\Sigma^{\prime}\right)^{G}=\mathcal{U}\left(\Sigma^{\prime}\right) \cap \mathcal{Q}\left[L^{*}\right]$

\section{Laurent phenomenon}

In what follows we restrict ourselves to the case $\operatorname{rank} L=2, \omega$ is a non-degenerate form and the vectors of exchange collection are primitive, however none of these conditions is essential.

Next theorem is the analogue of Theorem 1.5 in [3], presented here as Theorem A.1.

Theorem 3.1 (Laurent phenomenon in terms of upper bounds). Consider two C-seeds: $\Sigma=$ $\left(L, \omega ; v_{1}, \ldots, v_{n}\right)$ and $\Sigma^{\prime}=\left(L^{\prime}, \omega^{\prime} ; v_{1}^{\prime}, \ldots, v_{n}^{\prime}\right)$. If $\Sigma^{\prime}=\mu_{i} \Sigma$ is a mutation of $\Sigma$ in direction $1 \leqslant i \leqslant n$ then the upper bounds for $\Sigma$ and $\Sigma^{\prime}$ coincide: $\mathcal{U}(\Sigma)=\mu_{v_{i}}^{*} \mathcal{U}\left(\Sigma^{\prime}\right)$. As a corollary, if a seed $\Sigma^{\prime}$ is obtained from a seed $\Sigma$ by a sequence of mutations, then the upper bound $\mathcal{U}\left(\Sigma^{\prime}\right)$ equals to the upper bound $\mathcal{U}(\Sigma)$ under identification of the ambient field by composition of the birational mutations.

By Proposition 2.1 Theorem 3.1 is equivalent to the next corollary, which is easier to check in practice and has almost the same consequences as the main theorem of [9], presented here as Theorem B.1.

Corollary 3.1 ( $V$-lemma). If $V$-seeds $\Sigma$ and $\Sigma^{\prime}$ are related by a mutation then the seed $\Sigma$ satisfies property $(V) \Longleftrightarrow$ the seed $\Sigma^{\prime}$ satisfies property $(V)$.

In the rest of this section we prove Theorem 3.1. Our proof is quite similar to that of $[3]^{5}$ : The set-theoretic argument reduces the problem to exchange collection $V$ with small number of vectors ( 1 or 2 ) without counting of multiplicities. Actually, when the collection $V$ has only one vector the equality of the upper bounds is obvious from the definitions. When the exchange collection consists of two base vectors one can explicitly compute the upper bounds and compare them. Finally, the case of two non-base non-collinear vectors is thanks to functoriality.

First of all, let us fix the notations. If the rank two lattice $L$ is generated by a pair of vectors $e_{1}$ and $e_{2}$, then the dual lattice $L^{*}=\operatorname{Hom}(L, \mathbb{Z})$ has the dual base $f_{1}, f_{2}$ determined by $\left(f_{i}, e_{j}\right)=\delta_{i, j}$. The form $\omega$ is uniquely determined by its value $k=\omega\left(e_{1}, e_{2}\right)$, and further we denote this isomorphism class of forms by $\omega_{k}$. We assume that $k \neq 0$, i.e. the form $\omega$ is non-degenerate ${ }^{6}$, by swapping $e_{1}$ and $e_{2}$ one can exchange $k$ to $-k$. A base $e_{i}$ of $L$ corresponds to a base $x_{i}=X^{f_{i}}$ of $\mathbb{Z}\left[L^{*}\right]$.

Lemma 3.1. Let $V$ be an exchange collection in $(L, \omega)$ and $\Sigma=(L, \omega ; V)$ be the respective seed.

1. If $V$ is empty, then obviously $\mathcal{U}(L, \omega ; V)=\mathcal{Q}\left[L^{*}\right]$.

2. Otherwise, let $V_{\alpha}$ be a set of exchange collections such that for any $v \in L$ we have $m_{V}(v)=$ $\max _{\alpha} m_{V_{\alpha}}(v)$. Then

$$
\mathcal{U}(V)=\cap_{\alpha} \mathcal{U}\left(V_{\alpha}\right) .
$$

3. In particular, if for a vector $v \in L$ we define $V_{v}=m_{V}(v) \times v$ to be an exchange collection that consists of a single vector $v$ with multiplicity $m_{V}(v)$ and $\Sigma_{v}=\left(L, \omega ; V_{v}\right)$ be the respective seed, then $\mathcal{U}(\Sigma)=\cap_{v \in L}\left(\mathcal{Q}\left[\mathbf{y}^{ \pm}\right] \cap \mathcal{Q}\left[\left(\mu_{v}^{*}\right)^{m_{V}(v)} \mathbf{y}^{ \pm}\right]\right)=\cap_{v \in L} \mathcal{U}\left(\Sigma_{v}\right)$. In other words, the upper bound of a $C$-seed $\Sigma=(L, \omega ; \mathbf{y}, V)$ can be expressed as the intersection of the upper bounds for its 1-vector subseeds.

\footnotetext{
${ }^{5}$ See Appendix A and Remark A.4 for the detailed comparison.

${ }^{6}$ If $k=0$ then $\omega=0$ and all mutations are trivial.
} 
4. Let $V$ consist of a vector $v_{1}$ with multiplicity $m_{+} \geqslant 1$, a vector $v_{2}=-v_{1}$ with multiplicity $m_{-} \geqslant 0$, and vectors $v_{k}(k \geqslant 3)$ that are non-collinear to $v_{1}$ with some multiplicities $m_{k} \geqslant 0$. Consider exchange subcollections $V_{0}=\left\{m_{+} \times v_{1}, m_{-} \times\left(-v_{1}\right)\right\}$ and $V_{k}=\left\{1 \times v_{1}\right.$, $\left.m_{k} \times v_{k}\right\}(k \geqslant 3)$. Then

$$
\mathcal{U}(V)=\mathcal{U}\left(V_{0}\right) \cap \mathcal{U}\left(V_{3}\right) \cap \mathcal{U}\left(V_{4}\right) \cap \cdots
$$

5. Let $V^{\prime}=\mu_{1} V$ be an exchange collection obtained by mutation of $V$ in $v_{1}$; it consists of vector $-v_{1}$ with multiplicity $m_{-}+1 \geqslant 1$, vector $v_{1}$ with multiplicity $m_{+}-1 \geqslant 0$ and vectors $v_{k}^{\prime}=\mu_{v_{1}} v_{k}(k \geqslant 3)$ with multiplicities $m_{k}$. Similarly to the previous step define $V_{0}^{\prime}=\left\{\left(m_{-}+1\right) \times\left(-v_{1}\right),\left(m_{+}-1\right) \times v_{1}\right\}$ and $V_{k}^{\prime}=\left\{1 \times\left(-v_{1}\right), m_{k} \times v_{k}^{\prime}\right\}(k \geqslant 3)$. Then

$$
\mathcal{U}\left(V^{\prime}\right)=\mathcal{U}\left(V_{0}^{\prime}\right) \cap \mathcal{U}\left(V_{3}^{\prime}\right) \cap \mathcal{U}\left(V_{4}^{\prime}\right) \cap \cdots
$$

6. Hence, to proof Theorem 3.1 it is necessary and sufficient to show that

$$
\mathcal{U}\left(V_{0}\right)=\mu_{v_{1}}^{*} \mathcal{U}\left(V_{0}^{\prime}\right) \quad \text { and } \quad \mathcal{U}\left(V_{k}\right)=\mu_{v_{1}}^{*} \mathcal{U}\left(V_{k}^{\prime}\right) \quad(\text { for all } k \geqslant 3) .
$$

We will prove these equalities in Proposition 3.3 and Lemma 3.3.

Proposition 3.1. Let $v_{1}, v_{2} \in L$ be a pair of vectors $v_{1}=a e_{1}+b e_{2}, v_{2}=c e_{1}+d e_{2}$ such that $a d-b c=1$. Consider the lattice $L^{\prime}$ with the base $e_{1}^{\prime}, e_{2}^{\prime}$ and the form $\omega^{\prime}\left(e_{1}^{\prime}, e_{2}^{\prime}\right)=\omega\left(v_{1}, v_{2}\right)$; let $f_{1}^{\prime}, f_{2}^{\prime}$ be the dual base of $L^{\prime *}$. Consider a map $m: L \rightarrow L^{\prime}$ given by $m\left(e_{1}\right)=d e_{1}^{\prime}-b e_{2}^{\prime}, m\left(e_{2}\right)=$ $-c e_{1}^{\prime}+a e_{2}^{\prime}$; note that $m\left(v_{1}\right)=m\left(a e_{1}+b e_{2}\right)=e_{1}^{\prime}$ and $m\left(v_{2}\right)=m\left(c e_{1}+d e_{2}\right)=e_{2}^{\prime}$. The dual isomorphism $m^{*}: L^{* *} \rightarrow L^{*}$ is given by the transposed map $m^{*}\left(f_{1}^{\prime}\right)=d f_{1}-c f_{2}$ and $m^{*}\left(f_{2}^{\prime}\right)=-b f_{1}+a f_{2}$. Let $z_{1}=X^{f_{1}^{\prime}}=x_{1}^{d} x_{2}^{-c}$ and $z_{2}=X^{f_{2}^{\prime}}=x_{1}^{-b} x_{2}^{a}$. Since map $m^{*}$ is invertible by Proposition 2.2 it gives the equality

$$
\mathcal{U}\left(L, \omega ; m_{1} \times v_{1}, m_{2} \times v_{2}\right)=\left.\mathcal{U}\left(L^{\prime}, \omega^{\prime} ; m_{1} \times e_{1}^{\prime}, m_{2} \times e_{2}^{\prime}\right)\right|_{z_{1}=x_{1}^{d} x_{2}^{-c}, z_{2}=x_{1}^{-b} x_{2}^{a}} .
$$

Lemma 3.2. Assume a seed $\Sigma=\left(L, \omega ; m_{1} \times v_{1}\right)$ consists of a unique vector $v_{1}$ with multiplicity $m_{1} \geqslant 1$.

1. If $v_{1}=e_{2}=(0,1)$ then the upper bound $\mathcal{U}(\Sigma)$ consists of all Laurent polynomials $W$ of the form $W=\sum_{l} c_{l}\left(x_{1}\right) x_{2}^{l}$ where $c_{l} \in \mathcal{Q}\left[x_{1}^{ \pm}\right]$and for $l \leqslant 0$ we have that $c_{l}$ is divisible by $\left(1+x_{1}^{k}\right)^{-m_{1} l}$. Moreover, $\mathcal{U}(\Sigma)=\mathcal{Q}\left[x_{1}^{ \pm}, x_{2}, \frac{1}{x_{2}^{\prime \prime}}=\frac{\left(1+x_{1}^{k}\right)^{m_{1}}}{x_{2}}\right]$.

2. If $v_{1}=a e_{1}+b e_{2}=(a, b)$ is an arbitrary primitive vector then $\mathcal{U}(\Sigma)=\mathcal{Q}\left[z^{ \pm}, z_{1}, \frac{\left(1+z^{k}\right)^{m_{1}}}{z_{1}}\right]$ where $z=\frac{x_{1}^{a}}{x_{2}^{b}}, z_{1}=x_{1}^{r} x_{2}^{s}$ and $(r, s) \in \mathbb{Z}^{2}$ satisfies $r b+s a=1$.

Proof. Recall that mutation in the direction $e_{2}$ is given by $x_{1}^{\prime}=x$ and $x_{2}^{\prime}=\frac{x_{2}}{1+x_{1}^{k}}$. Assume we have a Laurent polynomial $W=\sum_{l \in \mathbb{Z}} c_{l}\left(x_{1}\right) x_{2}^{l}$. Then $W$ can be expressed in terms of $x_{1}$ and $x_{2}^{\prime}$ as $W=\sum_{l} c_{l}\left(x_{1}\right)\left(1+x_{1}^{k}\right)^{l}\left(x_{2}^{\prime}\right)^{l}$. This function is a Laurent polynomial in terms of $\left(x_{1}, x_{2}^{\prime}\right) \Longleftrightarrow c_{l}\left(x_{1}\right)\left(1+x_{1}^{k}\right)^{l}$ is a Laurent polynomial of $x_{1}$ for all $l$. This is equivalent to $c_{l}$ being divisible by $\left(1+x_{1}^{k}\right)^{-l}$ for $l \leqslant 0$. Similarly if we do $m_{1}$ mutations then $x_{2}^{\prime \prime}=\frac{x_{2}}{\left(1+x_{1}^{k}\right)^{a}}$ and $W=\sum c_{l}\left(1+x_{1}^{k}\right)^{m_{1} l}\left(x_{2}^{\prime \prime}\right)^{l}$ so for $l \leqslant 0$ we have that $c_{l}$ is divisible by $\left(1+x_{1}^{k}\right)^{-m_{1} l}$. Let $c_{l}\left(x_{1}\right)=$ $\left(1+x_{1}^{k}\right)^{-m_{1} l} c_{-l}^{\prime}\left(x_{1}\right)$ for $l<0, c_{l}^{\prime}$ are also Laurent polynomials. Denote $W_{+}=\sum_{l \geqslant 0} c_{l}\left(x_{1}\right) x_{2}^{l}$ and $W_{-}=\sum_{l<0} c_{l}\left(x_{1}\right) x_{2}^{l}=\sum_{l>0} c_{l}^{\prime}\left(x_{1}\right)\left(x_{2}^{\prime \prime}\right)^{-l}$. Then obviously both $W_{+}$and $W_{-}$belong to $\mathcal{Q}\left[x_{1}^{ \pm}, x_{2}, \frac{1}{x_{2}^{\prime \prime}}\right]$. The reverse inclusion is straightforward.

Part (2) follows from Proposition 3.1. 
Proposition 3.2. Let exchange collection $V$ consists of a vector $v_{1}=e_{2}=(0,1)$ with multiplicity $m_{1} \geqslant 0$ and its inverse $v_{2}=-v_{1}=-e_{2}=(0,-1)$ with multiplicity $m_{2} \geqslant 0$,

1. The upper bound $\mathcal{U}\left(L, \omega ; m_{1} \times e_{2}, m_{2} \times\left(-e_{2}\right)\right)$ consists of all Laurent polynomials $W$ of the form $W=\sum_{l} c_{l}\left(x_{1}\right) x_{2}^{l}$ where $c_{l} \in \mathcal{Q}\left[x_{1}^{ \pm}\right]$and for $l \leqslant 0$ we have that $c_{l}$ is divisible by $\left(1+x_{1}^{k}\right)^{-m_{1} l}$ and for $l \geqslant 0$ we have that $c_{l}$ is divisible by $\left(1+x_{1}^{k}\right)^{m_{2} l}$.

2. $\mathcal{U}\left(L, \omega ; m_{1} \times e_{2}, m_{2} \times\left(-e_{2}\right)\right)=\mathcal{Q}\left[x_{1}^{ \pm}, x_{2}\left(1+x_{1}^{k}\right)^{m_{2}}, \frac{\left(1+x_{1}^{k}\right)^{m_{1}}}{x_{2}}\right]$.

Proof. The first statement is a straightforward corollary of Lemmas 3.1 and 3.2(1). The proof of the second statement is similar to the end of the proof of Lemma 3.2(2): separate the Laurent polynomial $W$ into positive and negative parts $W_{+}$and $W_{-}$; then both parts lie in the ring $\mathcal{Q}\left[x_{1}^{ \pm}, x_{2}\left(1+x_{1}^{k}\right)^{m_{2}}, \frac{\left(1+x_{1}^{k}\right)^{m_{1}}}{x_{2}}\right]$.

Proposition 3.3. Assume a seed $\Sigma$ consists of a vector $v_{1}=(0,1)$ with multiplicity $m_{1}$ and its inverse $-v_{1}=(0,-1)$ with multiplicity $m_{2}$. Then its mutation $\Sigma^{\prime}=\mu_{1}(\Sigma)$ consists of $v_{1}$ and $-v_{1}$ with respective multiplicities $m_{1}-1$ and $m_{2}+1$. Then $\mathcal{U}(\Sigma)=\mathcal{U}\left(\Sigma^{\prime}\right)$.

Proof. By Proposition 3.2 the upper bounds are expressed as: $\mathcal{U}(\Sigma)=\mathcal{Q}\left[x_{1}^{ \pm}, x_{2}\left(1+x_{1}^{k}\right)^{m_{2}}\right.$, $\left.\frac{\left(1+x_{1}^{k}\right)^{m_{1}}}{x_{2}}\right], \mathcal{U}\left(\Sigma^{\prime}\right)=\mathcal{Q}\left[x_{1}^{\prime \pm}, \frac{\left(1+x_{1}^{\prime k}\right)^{m_{1}-1}}{x_{2}^{\prime}}, x_{2}^{\prime}\left(1+x_{1}^{\prime k}\right)^{m_{2}+1}\right]$. Since $x_{1}^{\prime}=x_{1}$ and $x_{2}^{\prime}=\frac{x_{2}}{1+x_{1}^{k}}$ we have the desired equality of the upper bounds.

Proposition 3.4. Assume that the seed $\Sigma=\left(L, \omega ; m_{1} \times v_{1}, m_{2} \times v_{2}\right)$ consists of vectors $v_{1}$ with multiplicity $m_{1} \geqslant 0$ and $v_{2}$ with multiplicity $m_{2} \geqslant 0$.

1. If $v_{1}=e_{1}$ and $v_{2}=e_{2}$, then the upper bound $\mathcal{U}(\Sigma)$ equals $\mathcal{Q}\left[x_{1}, x_{2}, \frac{\left(1+x_{2}^{k}\right)^{m_{1}}}{x_{1}}, \frac{\left(1+x_{1}^{k}\right)^{m_{2}}}{x_{2}}\right]$.

2. If $v_{1}=a e_{1}+b e_{2}$ and $v_{2}=c e_{1}+d e_{2}$ with $a d-b c=1$ then the upper bound $\mathcal{U}(\Sigma)$ equals $\mathcal{Q}\left[z_{1}, z_{2}, \frac{\left(1+z_{2}^{k}\right)^{m_{1}}}{z_{1}}, \frac{\left(1+z_{1}^{k}\right)^{m_{2}}}{z_{2}}\right]$ with $z_{1}=x_{1}^{d} x_{2}^{-c}$ and $z_{2}=x_{1}^{-b} x_{2}^{a}$.

Proof. For the first case, by Lemmas 3.1 and 3.2 we have $\mathcal{U}(\Sigma)=\mathcal{Q}\left[x_{1}^{ \pm}, x_{2}, \frac{\left(1+x_{1}^{k}\right)^{m_{2}}}{x_{2}}\right] \cap$ $\mathcal{Q}\left[x_{2}^{ \pm}, x_{1}, \frac{\left(1+x_{2}^{k}\right)^{m_{1}}}{x_{1}}\right]$. If $m_{1}=m_{2}=1$, by Proposition 4.3 of [3] (with $\left|b_{12}\right|=\left|b_{21}\right|=b=c=k$ and $\left.q_{1}=q_{2}=r_{1}=r_{2}=1\right)$ this intersection equals $\mathcal{Q}\left[x_{1}, x_{2}, \frac{1+x_{2}^{k}}{x_{1}}, \frac{1+x_{1}^{k}}{x_{2}}\right]$. Lemma 3.2 covers cases with $m_{1}=0$ or $m_{2}=0$. If $m_{1}$ and $m_{2}$ are greater than 1, the proof of Proposition 4.3 in [3] can be easily modified to include the case we need since $x_{2} \frac{1+x_{1}^{k}}{x_{2}}\left(1+x_{1}^{k}\right)^{m_{2}-1}=\left(1+x_{1}^{k}\right)^{m_{2}}$ and $x_{1} \frac{1+x_{2}^{k}}{x_{1}}\left(1+x_{2}^{k}\right)^{m_{1}-1}=\left(1+x_{2}^{k}\right)^{m_{1}}$, then the intersection equals $\mathcal{Q}\left[x_{1}, x_{2}, \frac{\left(1+x_{2}^{k}\right)^{m_{1}}}{x_{1}}, \frac{\left(1+x_{1}^{k}\right)^{m_{2}}}{x_{2}}\right]$.

Part (2) follows from Proposition 3.1.

Lemma 3.3. Let $\Sigma=\left(L, \omega ; 1 \times v_{1}, m_{2} \times v_{2}\right)$ be a seed of two non-collinear vectors $v_{1}$ and $v_{2}$ with $m\left(v_{1}\right)=1$ and $m\left(v_{2}\right)=m_{2} \geqslant 0$ and $\Sigma^{\prime}=\Sigma_{1}=\left(L^{\prime}=L, \omega^{\prime}=\omega ; v_{1}^{\prime}=-v_{1}, m_{2} \times\left(v_{2}^{\prime}=\mu_{v_{1}} v_{2}\right)\right)$ be the mutation of the seed $\Sigma$ in $v_{1}$. Then $\mathcal{U}(\Sigma)=\mu_{v_{1}}^{*} \mathcal{U}\left(\Sigma^{\prime}\right){ }^{7}$

Proof. First of all note that, $\omega^{\prime}\left(v_{1}^{\prime}, v_{2}^{\prime}\right)=-\omega\left(v_{1}, v_{2}\right)$ and since $\mu_{-v_{1}} \mu_{v_{1}}=R_{v_{1}}$ it is sufficient to consider only the case $\omega\left(v_{1}, v_{2}\right)>0$. We first consider the case when $v_{1}=e_{1}=(1,0)$ and $v_{2}=e_{2}=(0,1)$; denote $k=\omega\left(e_{1}, e_{2}\right)>0$. Let $e_{1}^{\prime}, e_{2}^{\prime}$ be the base of $L^{\prime}$ that corresponds to $e_{1}, e_{2}$ under the natural identification of $L^{\prime} \simeq L$; finally consider a base $e_{1}^{\prime \prime}, e_{2}^{\prime \prime}$ of $L^{\prime}$ given by $e_{1}^{\prime \prime}=v_{2}^{\prime}=k e_{1}^{\prime}+e_{2}^{\prime}, e_{2}^{\prime \prime}=v_{1}^{\prime}=-e_{1}^{\prime}$. Let $f_{1}^{\prime}, f_{2}^{\prime}$ and $f_{1}^{\prime \prime}, f_{2}^{\prime \prime}$ be the respective dual bases of $L^{\prime *}$. Thus we have one natural regular system of coordinates $x_{1}=X^{f_{1}}, x_{2}=X^{f_{2}}$ on the torus $T=$

\footnotetext{
${ }^{7}$ Denote $\Sigma_{2}=\left\{\left(\mu_{v_{2}}\right)^{m_{2}} v_{1},-v_{2}\right\}-m_{2}$-multiple mutation of $\Sigma$ in $v_{2}$, and $\Sigma_{2}^{\prime}=\left\{\left(\mu_{v_{2}^{\prime}}\right)^{m_{2}} v_{1}^{\prime},-v_{2}^{\prime}\right\}-m_{2}$-multiple mutation of $\Sigma^{\prime}$ in $v_{2}^{\prime}$. We are going to prove that $\mathcal{U}(\Sigma)=\mathcal{Q}[\mathbf{y}] \cap \mathcal{Q}\left[\mathbf{y}^{\prime}=\mathbf{y}_{\mathbf{1}}\right] \cap \mathcal{Q}\left[\mathbf{y}_{\mathbf{2}}\right]$ equals to $\mathcal{U}\left(\Sigma^{\prime}\right)=\mathcal{Q}[\mathbf{y}] \cap \mathcal{Q}\left[\mathbf{y}^{\prime}=\right.$ $\left.\mathbf{y}_{\mathbf{1}}\right] \cap \mathcal{Q}\left[\mathbf{y}_{\mathbf{2}}^{\prime}\right]$.
} 
Spec $\mathbb{Z}\left[L^{*}\right]$, and two regular systems of coordinates $x_{1}^{\prime}=X^{f_{1}^{\prime}}, x_{2}^{\prime}=X^{f_{2}^{\prime}} ; x_{1}^{\prime \prime}=X^{f_{1}^{\prime \prime}}, x_{2}^{\prime \prime}=X^{f_{2}^{\prime \prime}}$ on the torus $T^{\prime}=\operatorname{Spec} \mathbb{Z}\left[L^{\prime *}\right]$. Taking $a=k, b=1, c=-1$ and $d=0$ in Proposition 3.1 we have that $x_{1}^{\prime \prime}=x_{2}^{\prime}$ and $x_{2}^{\prime \prime}=\frac{x_{2}^{\prime k}}{x_{1}^{\prime}}$, since $x_{2}^{\prime}=x_{2}$ and $x_{1}^{\prime}=\frac{x_{1} x_{2}^{k}}{1+x_{2}^{k}}$ (they are the mutations of $x_{1}$ and $x_{2}$ with respect to $v_{1}$ ), thus what we need to show is that the rings $\mathcal{Q}\left[x_{1}, x_{2}, \frac{1+x_{2}^{k}}{x_{1}}, \frac{1+x_{1}^{k}}{x_{2}}\right]$ and $\mathcal{Q}\left[x_{1}, x_{2}, \frac{1+x_{2}^{k}}{x_{1}}, \frac{x_{1}^{k}+\left(1+x_{2}^{k}\right)^{k}}{x_{1}^{k} x_{2}}\right]$ are equal. We will first show that $\frac{x_{1}^{k}+\left(1+x_{2}^{k}\right)^{k}}{x_{1}^{k} x_{2}} \in \mathcal{Q}\left[x_{1}, x_{2}, \frac{1+x_{2}^{k}}{x_{1}}, \frac{1+x_{1}^{k}}{x_{2}}\right]$. We have that $\frac{x_{1}^{k}+\left(1+x_{2}^{k}\right)^{k}}{x_{1}^{k} x_{2}}=\left(\frac{1+x_{1}^{k}}{x_{2}}\right)\left(\frac{\left(1+x_{2}^{k}\right)^{k}}{x_{1}^{k}}\right)-\sum_{j=1}^{k} \frac{k !}{j !(k-j) !} x_{2}^{k j-1}$. Clearly the expression in the right side belongs to $\mathcal{Q}\left[x_{1}, x_{2}, \frac{1+x_{2}^{k}}{x_{1}}, \frac{1+x_{1}^{k}}{x_{2}}\right]$. Now, we will show that $\frac{1+x_{1}^{k}}{x_{2}} \in \mathcal{Q}\left[x_{1}, x_{2}, \frac{1+x_{2}^{k}}{x_{1}}, \frac{x_{1}^{k}+\left(1+x_{2}^{k}\right)^{k}}{x_{1}^{k} x_{2}}\right]$. We have that $\frac{1+x_{1}^{k}}{x_{2}}=x_{1}^{k} \frac{x_{1}^{k}+\left(1+x_{2}^{k}\right)^{k}}{x_{1}^{k} x_{2}}-\sum_{j=1}^{k} \frac{k !}{j(k-j) !} x_{2}^{k j-1}$. Again, clearly the expression in the right side belongs to $\mathcal{Q}\left[x_{1}, x_{2}, \frac{1+x_{2}^{k}}{x_{1}}, \frac{x_{1}^{k}+\left(1+x_{2}^{k}\right)^{k}}{x_{1}^{k} x_{2}}\right]$. Thus, we have the equality between the rings. Similarly, if the multiplicity of $v_{2}$ is $m_{2}>1$, we have that $\mathcal{Q}\left[x_{1}, x_{2}, \frac{\left(1+x_{2}^{k}\right)}{x_{1}}, \frac{\left(1+x_{1}^{k}\right)^{m_{2}}}{x_{2}}\right]=$ $\mathcal{Q}\left[x_{1}, x_{2}, \frac{1+x_{2}^{k}}{x_{1}}, \frac{\left(x_{1}^{k}+\left(1+x_{2}^{k}\right)^{k}\right)^{m_{2}}}{x_{1}^{m_{2}} x_{2}}\right] .{ }^{8}$ If $v_{1}$ and $v_{2}$ are another basis of $\mathbb{Z}^{2}$ the result follows from Proposition 3.1. In case $v_{1}$ and $v_{2}$ is a pair of non-collinear vectors which are not a basis for $\mathbb{Z}^{2}$, consider the sublattice $L^{\prime} \subset L$ generated by $e_{1}^{\prime}=v_{1}$ and $e_{2}^{\prime}=v_{2}$ with the form $\omega^{\prime}=\left.\omega\right|_{L^{\prime}}$. As we just saw upper bounds with respect to the sublattice coincide: $\mathcal{U}\left(L^{\prime}, \omega^{\prime} ; v_{1}, m_{2} \times v_{2}\right)=$ $\mu_{v_{1}}^{*} \mathcal{U}\left(L^{\prime}, \omega^{\prime} ;-v_{1}, m_{2} \times \mu_{v_{1}} v_{2}\right)$. Now the statement follows from the Proposition 2.3.

Remark 3.1. If a mutation of a Laurent polynomial with integer coefficients happened to be a Laurent polynomial, then its coefficients are also integer. Let $u \in L$ be a primitive vector and $W, W^{\prime} \in \mathcal{Q}\left[L^{*}\right]$ be a pair of Laurent polynomials with arbitrary coefficients such that $W=\mu_{u}^{*} W^{\prime}$. Then $W \in \mathbb{Z}\left[L^{*}\right] \Longleftrightarrow W^{\prime} \in \mathbb{Z}\left[L^{*}\right]$.

Proof. Choose coordinates on $L$ so that $u=e_{2}$. Assume $W$ has integer coefficients. By Lemma 3.2(1) $W=\sum c_{l}\left(x_{1}\right) x_{2}^{l}$ and $W^{\prime}=\sum_{l \in \mathbb{Z}} c_{l}^{\prime}\left(x_{1}\right) x_{2}^{\prime l}$ with $c_{l}^{\prime}=c_{l}\left(1+x_{1}^{k}\right)^{-l}$ for all $l \in \mathbb{Z}$. Clearly $W \in \mathbb{Z}\left[L^{*}\right] \Longleftrightarrow$ all coefficients of $W$ are integer $\Longleftrightarrow$ for all $l \in \mathbb{Z}$ all coefficients of $c_{l}$ are integer. Since $\left(1+x_{1}^{k}\right) \in \mathbb{Q}\left[x_{1}^{ \pm}\right]$it is clear that $c_{l}^{\prime} \in \mathbb{Q}\left[x_{1}^{ \pm}\right]$. Recall that for a Laurent polynomial $P \in \mathbb{Q}[x]$ its Gauss's content $C(P) \in \mathbb{Q}$ is defined as the greatest common divisor of all its coefficients: if $P=\sum a_{i} x^{i}$ then $C=\operatorname{gcd}\left(a_{i}\right)$. Clearly $C(P) \in \mathbb{Z} \Longleftrightarrow P \in \mathbb{Z}\left[x^{ \pm}\right]$. Gauss's lemma says that $C\left(P \cdot P^{\prime}\right)=C(P) \cdot C\left(P^{\prime}\right)$. Since $C\left(1+x_{1}^{k}\right)=\operatorname{gcd}(1,1)=1$ we see that $C\left(c_{l}^{\prime}\right)=C\left(c_{l}\right) \cdot 1^{-l}=C\left(c_{l}\right)$, hence $c_{l}^{\prime} \in \mathbb{Z}\left[x_{1}^{ \pm}\right] \Longleftrightarrow c_{l} \in \mathbb{Z}\left[x_{1}^{ \pm}\right]$.

\section{Questions and future developments}

In the introduction was pointed out that our definition of upper bounds makes plausible to consider a quantum version of mutations of potentials and the corresponding quantum Laurent phenomenon. On the other hand, in [10] a non-commutative version of the Laurent phenomenon is discussed. Thus, we would like to ask:

Question 4.1. Is it possible to consider a non-commutative version of the Laurent phenomenon for mutation of potentials and develop a theory of upper bounds in this context?

In [9] the following problem (Problem 44) was proposed

Question 4.2. Construct a fiberwise-compact canonical mirror of a Fano variety as a gluing of open charts given by (all) different toric degenerations.

\footnotetext{
${ }^{8}$ The argument for showing the equality of these two rings is the same of that when $m_{2}=1$, but the computations are slightly longer, so we omit them.
} 
Conjecture 4.1 (which will be proved in [5]) gives a partial answer for the above question.

Conjecture 4.1. For the 10 potentials $W$ (i.e., $\left(W_{1}, W_{2}, \ldots, W_{9}, W_{Q}\right)$ ) listed in [9] (or rather the exchange collections $V$ (resp. $\left.V_{1}, \ldots, V_{9}, V_{Q}\right)$ ) the upper bound $\mathcal{U}(V)$ is the algebra of polynomials in one variable. Moreover, this variable is $W$.

Conjecture 4.1 is useful for symplectic geometry as long as one knows two (non-trivial) properties of the FOOO's potentials $m_{0}$ [8] (here $W=m_{0}$ ):

1. $W$ is a Laurent polynomial (this is some kind of convergence/finiteness property).

2. $W$ is transformed according to Auroux's wall-crossing formula [2], and more specifically by the mutations described in Section 2. The directions of the mutations/walls are encoded by an exchange collection $V$.

What we believe is that once one knows these assumptions, one should be able to prove that some disc-counting potential equals some particularly written $W$ (formally) without any actual disc counting. Needless to say this is a speculative idea.

\section{A Review of the classical cluster algebras, upper bounds and Laurent phenomenon}

In this appendix we review some results of the first section of [3]: approach to Laurent phenomenon via upper bounds by Berenstein, Fomin and Zelevinsky, and make a brief comparison between their theory and the one presented here. We will denote the framework of cluster algebras developed by Berenstein, Fomin and Zelevinsky in [3] by BFZ.

\section{A.1 Definitions of exchange matrix, coefficients, cluster and seed}

Fix $n$-dimensional lattice $L \simeq \mathbb{Z}^{n}$. The underlying combinatorial gadget in the theory of cluster algebras is a $n \times n$ matrix.

Definition A.1 (exchange matrix $B$ ). An exchange matrix is a sign-skew-symmetric $n \times n$ integer matrix $B=\left(b_{i j}\right)$ : for any $i$ and $j$, either $b_{i j}=b_{j i}=0$ or $b_{i j} b_{j i}<0$.

Obviously a skew-symmetric matrix is sign-skew-symmetric, and for simplicity we assume further that $B$ is skew-symmetric.

Any matrix $B$ can be considered as an element of $L^{*} \otimes L^{*}$. Skew-symmetric matrices are then identified with $\wedge^{2}(L)$.

Let $\mathbb{P}$ be the coefficient group - an Abelian group without torsion written multiplicatively. Fix an ambient field $\mathbb{F}$ of rational functions on $n$ independent variables with coefficients in (the field of fractions of) the integer group ring $\mathbb{Z P}$.

Definition A.2 (coefficients). A coefficient tuple $\mathbf{p}$ is an $n$-tuple of pairs $\left(p_{i}^{+}, p_{i}^{-}\right) \in \mathbb{P}^{2}$.

Finally the non-combinatorial object of the theory is a cluster.

Definition A.3 (BFZ-cluster). A cluster $\mathbf{x}=\left(x_{1}, \ldots, x_{n}\right)$ is a transcendence basis of $\mathbb{F}$ over the field of fractions of $\mathbb{Z} \mathbb{P}$. Let $\mathbb{Z} \mathbb{P}\left[\mathbf{x}^{ \pm 1}\right]$ denote the ring of Laurent polynomials of $x_{1}, \ldots, x_{n}$ with coefficients in $\mathbb{Z P}$.

Definition A.4 (BFZ-seed). A seed (or BFS-seed) is a triple ( $\mathbf{x}, \mathbf{p}, B)$ of a cluster, coefficients tuple and exchange matrix.

Remark A.1 (action of the symmetric group $S_{n}$ ). As noticed in [3] the symmetric group $S_{n}$ naturally acts on exchange matrices, coefficients, clusters, and hence seeds by permutating indices $i$. 


\section{A.2 Mutations}

For each $1 \leqslant k \leqslant n$ we can define the mutation of exchange matrix $B$, of a pair $(B, \mathbf{p})$ and of a seed $(B, \mathbf{p}, \mathbf{x})$.

Definition A.5 (mutation $\mu_{i}$ of an exchange matrix $\left.B\right)$. Given an exchange matrix $B=\left(b_{i j}\right)$ and an index $1 \leqslant k \leqslant n$ define $\mu_{k} B=B^{\prime}=\left(b_{i j}^{\prime}\right)$ as follows: $b_{i k}^{\prime}=-b_{i k}, b_{k j}^{\prime}=-b_{k j}$, and otherwise $b_{i j}^{\prime}=b_{i j}+\frac{\left|b_{i k}\right| b_{k j}+b_{i k}\left|b_{k j}\right|}{2}$.

It is easy to check that $\mu_{k}\left(\mu_{k}(B)\right)=B$.

Definition A.6 (mutations of coefficients). Given an exchange matrix $B$ and a coefficients tuple $\mathbf{p}$ define a mutation of the coefficients in direction $k$ as any new $n$-tuple $\left(p_{i}^{\prime+}, p_{i}^{\prime-}\right)$ that satisfies $\frac{p_{i}^{\prime+}}{p_{i}^{\prime-}}=\left(p_{k}^{+}\right)^{b_{k i}} \frac{p_{i}^{+}}{p_{i}^{-}}$if $b_{k i} \geqslant 0$ and $\frac{p_{i}^{\prime+}}{p_{i}^{\prime-}}=\left(p_{k}^{-}\right)^{b_{k i}} \frac{p_{i}^{+}}{p_{i}^{-}}$if $b_{k i} \leqslant 0$.

In this definition the choice of a new $n$-tuple has $(n-1)$ degrees of freedom. This ambiguity is not important, however one of the ways of curing this ambiguity is by considering tuples with $p^{-}=1$. Also one can get rid of coefficients by considering the trivial tuples $p^{+}=p^{-}=1$.

Definition A.7 (mutations of seeds). The mutation of a seed $\Sigma=(\mathbf{x}, \mathbf{p}, B)$ in the direction $1 \leqslant k \leqslant n$ is a new seed $\Sigma^{\prime}=\left(\mathbf{x}_{k}, \mathbf{p}^{\prime}, B^{\prime}\right)$ where $B^{\prime}=\mu_{k} B$ is a mutation of the exchange matrix $B$ in the direction $k, \mathbf{p}^{\prime}$ is a mutation of $\mathbf{p}$ using $B$ in the direction $k$ (Definition A.6), and $\mathbf{x}^{\prime}$ is defined as follows: $x_{k}^{\prime} x_{k}=P_{k}(\mathbf{x})=p_{j}^{+} \prod_{b_{i k}>0} x_{i}^{b_{i k}}+p_{k}^{-} \prod_{b_{i k}<0} x_{i}^{-b_{i k}}$ and $x_{i}^{\prime}=x_{i}$ for $i \neq k$.

The next definition is a technicality required by [3] for the proof.

Definition A.8. A seed $\Sigma$ is called coprime if polynomials $P_{1}, \ldots, P_{n}$ are pairwise coprime in $\mathbb{Z} \mathbb{P}[\mathbf{x}]$.

\section{A.3 Upper bounds and Laurent phenomenon}

Definition A.9 (upper bound $\mathcal{U}(\Sigma)$ ). For a BFZ-seed $\Sigma$ its upper bound is the $\mathbb{Z} \mathbb{P}$-subalgebra of $\mathbb{F}$ given by

$$
\mathcal{U}(\Sigma)=\mathbb{Z} \mathbb{P}\left[\mathbf{x}^{ \pm 1}\right] \cap \mathbb{Z} \mathbb{P}\left[\mathbf{x}_{\mathbf{1}}^{ \pm 1}\right] \cap \cdots \cap \mathbb{Z} \mathbb{P}\left[\mathbf{x}_{\mathbf{n}}{ }^{ \pm 1}\right]
$$

The next theorem is a manifestation of the Laurent phenomenon in terms of upper bounds.

Theorem A.1 ([3, Theorem 1.5]). If two seeds $\Sigma$ and $\Sigma^{\prime}$ are related by a seed mutation and both are coprime, then the corresponding upper bounds coincide: $\mathcal{U}(\Sigma)=\mathcal{U}\left(\Sigma^{\prime}\right)$.

\section{A.4 Relations between BFZ with [9] and this paper}

Given an exchange collection $V=\left(v_{1}, \ldots, v_{n}\right)$ one can associate a skew-symmetric $n \times n$ matrix $B(V)=\left(b_{i j}\right)$ :

$$
b_{i, j}=\omega\left(v_{i}, v_{j}\right) .
$$

Lemma A.1. For any $V$ and $1 \leqslant k \leqslant n$ we have $B\left(\mu_{k} V\right)=\mu_{k} B(V)$.

Proof. Indeed, let $B(V)=\left(b_{i j}\right)$ and $B\left(\mu_{k} V\right)=\left(b_{i j}^{\prime}\right)$. Then $b_{i j}^{\prime}=\omega\left(v_{i}+\max \left(0, \omega\left(v_{k}, v_{i}\right)\right) v_{k}, v_{j}+\right.$ $\left.\max \left(0, \omega\left(v_{k}, v_{j}\right)\right) v_{k}\right)=\omega\left(v_{i}, v_{j}\right)+\max \left(0, \omega\left(v_{k}, v_{i}\right)\right) \omega\left(v_{k}, v_{j}\right)+\max \left(0, \omega\left(v_{k}, v_{j}\right)\right) \omega\left(v_{i}, v_{k}\right)=b_{i j}+$ $\max \left(0,-b_{i k}\right) b_{k j}+\max \left(0, b_{k j}\right) b_{i k}=b_{i j}+a \cdot b_{i k} b_{k j}$, where $a=\frac{\operatorname{sgn}\left(b_{i k}\right)+\operatorname{sgn}\left(b_{k j}\right)}{2}$, i.e. 1 if both $b_{i k}$ and $b_{k j}$ are positive, -1 if they are both negative, and 0 otherwise. It is easy to check that this coincides with Definition A.5. 
Remark A.2. We note that in case $\operatorname{rank} L=2$ the matrix $B(V)$ is a very special skew-symmetric matrix: it is non-zero only if the collection $V$ has at least two non-collinear vectors, and in this case its rank equals two.

Remark A.3. For an exchange collection $V \in L^{n}$ the sublattice $L_{V}$ in $L$ denotes the sublattice generated by $v_{i}$. It can be seen that $L_{V}$ is preserved under mutations of $V$, and actually can be reconstructed from $B(V)$ if $\omega$ is non-degenerate on $L_{V}$.

Remark A.4. Roughly the setup of BFZ corresponds to a special class of $C$-seeds with $v_{1}, \ldots, v_{n}$ being a base of the lattice $L$ with all multiplicities equal to 1 . Thus the proof of Theorem 3.1 mostly reduces to Theorem A.1 and its proof, with extra care of keeping track of all the multiplicities and exploiting nice functorial properties with respect to the maps of the lattices and subcollections.

Lemmas 3.1 and 3.2 are analogues and almost immediate consequences of Lemmas 4.1 and 4.2 in [3]. Propositions 3.2 and 3.3 are analogues of Case 1 in the proof of Proposition 4.3 in [3]. Proposition 3.4 is analogue of the Case 2 in the proof of Proposition 4.3 of [3]. Lemma 3.3 is similar to Lemma 4.6 in [3].

\section{B Definitions from [9]}

Definition B.1 ( $U$-seed). A $U$-seed is a quadruple $(W, V, F, X)$ where $V \in L_{1}^{n}$ is an exchange collection, $F$ is a fan in $M, X$ is a toric surface associated with the fan $F$ and $W$ is a rational function on $X$. In addition, given a $U$-seed we can define a curve $C$ by the equation

$$
C-\Sigma_{t} n_{t} D_{t}=(W),
$$

where $\Sigma_{t} n_{t} D_{t}$ is the part corresponding to toric divisors.

Definition B.2 (property $(U)$ ). We say a $U$-seed satisfies property $(U)$ if the following conditions hold:

1) $C$ is an effective divisor, i.e. $W$ is a Laurent polynomial;

2) $C=A+B$, where $A$ is an irreducible non-rational curve and $B$ is supported on rational curves;

3) the intersection of $C$ with toric divisors has canonical coordinates -1 ;

4) if $t \in V$, then the intersection index $\left(C \cdot D_{t}\right) \geqslant n_{t}$;

$5)$ for a toric divisor $D_{t}$ the intersection index $\left(A \cdot D_{t}\right)$ equals the number of $i$ such that $v_{i}=t$.

In [9] the Laurent phenomenon is established in the following terms

Theorem B.1 (U-lemma). If two $U$-seeds $\Sigma$ and $\Sigma^{\prime}$ are related by a mutation then $\Sigma$ satisfies property $(U) \Longleftrightarrow \Sigma^{\prime}$ satisfies property $(U)$.

\section{Acknowledgements}

We want to thank Bernhard Keller for interesting discussions and in particular for suggesting the idea of considering upper bounds in the context of [9]. We thank Denis Auroux, Arkady Berenstein, Alexander Efimov, Alexander Goncharov and especially Alexandr Usnich for generously sharing their insights on mutations or mirror symmetry. We would like to thank Martin Guest for reading the draft of this paper and his valuable comments. We also want to thank the anonymous referees for their helpful comments and suggestions. First author was supported by a Japanese Government (Monbukagakusho:MEXT) Scholarship. Also this work was supported 
by World Premier International Research Center Initiative (WPI Initiative), MEXT, Japan, Grant-in-Aid for Scientific Research (10554503) from Japan Society for Promotion of Science, Grant of Leading Scientific Schools (N.Sh. 4713.2010.1), grants ERC GEMIS and FWF P 24572N25. The authors thank the IPMU for its hospitality and support during their visits in May and November 2012 which helped to finish this work.

\section{References}

[1] Akhtar M., Coates T., Galkin S., Kasprzyk A.M., Minkowski polynomials and mutations, SIGMA 8 (2012), 094, 707 pages, arXiv:1212.1785.

[2] Auroux D., Mirror symmetry and T-duality in the complement of an anticanonical divisor, J. Gökova Geom. Topol. GGT 1 (2007), 51-91, arXiv:0706.3207.

[3] Berenstein A., Fomin S., Zelevinsky A., Cluster algebras. III. Upper bounds and double Bruhat cells, Duke Math. J. 126 (2005), 1-52, math.RT/0305434.

[4] Berenstein A., Zelevinsky A., Quantum cluster algebras, Adv. Math. 195 (2005), 405-455, math.QA/0404446.

[5] Cruz Morales J.A., Galkin S., Quantized mutations of potentials and their upper bounds, in preparation.

[6] Fock V.V., Goncharov A.B., Cluster ensembles, quantization and the dilogarithm, Ann. Sci. Éc. Norm. Supér. (4) 42 (2009), 865-930, math.AG/0311245.

[7] Fomin S., Zelevinsky A., Cluster algebras. I. Foundations, J. Amer. Math. Soc. 15 (2002), 497-529, math.RT/0104151.

[8] Fukaya K., Oh Y.G., Ohta H., Ono K., Lagrangian Floer theory and mirror symmetry on compact toric manifolds, arXiv:1009.1648.

[9] Galkin S., Usnich A., Mutations of potentials, Preprint IPMU 10-0100, 2010.

[10] Kontsevich M., Noncommutative identities, arXiv:1109.2469.

[11] Zelevinsky A., Quantum cluster algebras: Oberwolfach talk, February 2005, math.QA/0502260. 